\title{
Excitatory/Inhibitory Responses Shape Coherent Neuronal Dynamics Driven by Optogenetic Stimulation in the Primate Brain
}

\author{
Ryan A. Shewcraft, ${ }^{1}$ Heather L. Dean, ${ }^{1}$ Margaret M. Fabiszak, ${ }^{2}{ }^{\oplus}$ Maureen A. Hagan, ${ }^{3,4}$ Yan T. Wong, ${ }^{3,4,5}$ \\ and ${ }^{\circ}$ Bijan Pesaran ${ }^{1,6}$ \\ ${ }^{1}$ Center for Neural Science, New York University, New York, New York 10003, ${ }^{2}$ Laboratory of Neural Systems, The Rockefeller University, New York, New \\ York 10065, ${ }^{3}$ Biomedicine Discovery Institute, ${ }^{4}$ Australian Research Council, Centre of Excellence for Integrative Brain Function, ${ }^{5}$ Department of Electrical \\ and Computer Systems Engineering, Monash University, Clayton, Victoria 3800, Australia, and ${ }^{6}$ New York University Neuroscience Institute, NYU \\ Langone Health, New York, New York 10016
}

Coherent neuronal dynamics play an important role in complex cognitive functions. Optogenetic stimulation promises to provide new ways to test the functional significance of coherent neural activity. However, the mechanisms by which optogenetic stimulation drives coherent dynamics remain unclear, especially in the nonhuman primate brain. Here, we perform computational modeling and experiments to study the mechanisms of optogenetic-stimulation-driven coherent neuronal dynamics in three male nonhuman primates. Neural responses arise from stimulation-evoked, temporally dynamic excitatory (E) and inhibitory (I) activity. Spiking activity is more likely to occur during E/I imbalances. Thus the relative difference in the driven E and I responses precisely controls spike timing by forming a brief time interval of increased spiking likelihood. Experimental results agree with parameter-dependent predictions from the computational models. These results demonstrate that optogenetic stimulation driven coherent neuronal dynamics are governed by the temporal properties of E/I activity. Transient imbalances in excitatory and inhibitory activity may provide a general mechanism for generating coherent neuronal dynamics without the need for an oscillatory generator.

Key words: balanced networks; neural coherence; nonhuman primate; optogenetic stimulation

Significance Statement

We examine how coherent neuronal dynamics arise from optogenetic stimulation in the primate brain. Using computational models and experiments, we demonstrate that coherent spiking and local field potential activity is generated by stimulationevoked responses of excitatory and inhibitory activity in networks, extending the growing literature on neuronal dynamics. These responses create brief time intervals of increased spiking tendency and are consistent with previous observations in the literature that balanced excitation and inhibition controls spike timing, suggesting that optogenetic-stimulation-driven coherence may arise from intrinsic E/I balance. Most importantly, our results are obtained in nonhuman primates and thus will play a leading role in driving the use of causal manipulations with optogenetic tools to study higher cognitive functions in the primate brain.

\section{Introduction}

Spiking and local field potential (LFP) activity display coherent patterns of activity across a range of temporal frequencies (Pesa-

Received Aug. 10, 2019; revised Jan. 10, 2020; accepted Jan. 14, 2020.

Author contributions: R.A.S., H.L.D., Y.T.W., and B.P. designed research; R.A.S., H.L.D., M.M.F., M.A.H., Y.T.W., and B.P. performed research; R.A.S. and B.P. contributed unpublished reagents/analytic tools; R.A.S. and B.P. analyzed data; R.A.S. and B.P. wrote the paper.

This work was supported, in part, by U.S. National Institutes of Health (NIH) BRAIN Initiative Grant R01NS104923, NIH R01-EY024067, NIH P30-EY013079, NIH Training Grant T32-EY007136, a Scholar Award from the McKnight Endowment Fund for Neuroscience (B.P.), and the Alfred P Sloan Foundation (B.P.).

The authors declare no competing financial interests.

Correspondence should be addressed to Bijan Pesaran at bijan@nyu.edu. ran et al., 2018). Coherent temporal structure has been proposed to play an important role in the control of higher cognitive functions (Womelsdorf et al., 2006; Buschman and Miller, 2007; Dean et al., 2012; Wong et al., 2016) and enhanced corticocortical communication more generally (Fries, 2005; Pesaran et al., 2018). Despite this, whether and how complex cognitive functions depend on the temporal structure of neuronal activity, as opposed to the magnitude of the activity, has been highly debated. Causal manipulations that generate frequency-selective coherent neuro- 
nal dynamics are important for directly testing their functional significance. However, to interpret the results of causal manipulations we also need to understand how extrinsic modulations of coherent neuronal dynamics recruit intrinsic cellular and network mechanisms to generate the driven response.

In general, coherent neuronal dynamics depend on the temporal precision and synchrony of spike timing (Buzsáki and Wang, 2012), which is governed by the intrinsic dynamics of excitatory (E) and inhibitory (I) postsynaptic potentials (Brunel and Wang, 2003; Wehr and Zador, 2003). Typically, excitatory and inhibitory activity are tightly correlated, with E preceding I by several milliseconds (Okun and Lampl, 2008). Thus, inputs that drive excitatory activity are closely followed by inhibitory activity and form a brief time interval during which excitation exceeds inhibition, allowing for temporally precise spiking in the postsynaptic neurons (Gabernet et al., 2005; Haider et al., 2013; Salkoff et al., 2015). The instantaneous frequency of coherent neuronal activity is regulated by $\mathrm{E} / \mathrm{I}$ balance (Atallah and Scanziani, 2009) and depends on the relative timing of $\mathrm{E}$ and I activity (Keeley et al., 2017; Chariker et al., 2018). Empirically observed neural activity is dynamic and episodic in the gamma (Burns et al., 2010, 2011; Xing et al., 2012) and beta (Feingold et al., 2015; Sherman et al., 2016; Rule et al., 2017) bands, suggesting that oscillatory E and I activity may not be necessary to generate coherent neuronal dynamics.

We tested the hypothesis that optogenetic stimulation can drive coherent neuronal dynamics by generating E/I responses that arise from intrinsic $\mathrm{E} / \mathrm{I}$ balance. Optogenetic stimulation has been shown to alter neuronal dynamics extrinsically by manipulating either excitatory or inhibitory activity alone (Cardin et al., 2009; Sohal et al., 2009; Adesnik and Scanziani, 2010; Lu et al., 2015; Ni et al., 2016; Veit et al., 2017). However, neuronal dynamics depend on the interaction between excitatory and inhibitory activity, which is governed by the local circuit. Because stimulating either excitatory or inhibitory activity alone recruits both excitatory and inhibitory postsynaptic responses in the network (Pike et al., 2000; Gloveli et al., 2005; Tukker et al., 2007; Buzsáki and Wang, 2012; Phillips and Hasenstaub, 2016), optogenetic stimulation may generate coherent neuronal dynamics according to the relative timing of driven excitatory and inhibitory responses.

We first developed a computational model of coherent neuronal dynamics in response to optogenetic stimulation. The individual $\mathrm{E}$ and I responses in the model have different dynamics and combine to generate a brief, phasic increase in the likelihood of spiking. The model shows that the coherent neuronal dynamics depend on the temporal properties of this combined E/I response, without the need for an oscillatory generator. We experimentally tested the model predictions by expressing ChR2(H134R) in macaque cortex delivered by an AAV2/5 viral injection and measuring spiking and LFP responses to sequences of optogenetic stimulation pulses. We demonstrate the generality of the findings across multiple regions of the posterior parietal and frontal cortex. Finally, we developed a spiking neural network model of the observed neural dynamics and found that $\mathrm{E} / \mathrm{I}$ responses with different dynamics are an emergent property of the model. Together these results suggest that optogenetic stimulation extrinsically alters excitatory and inhibitory activity by recruiting E/I responses to shape coherent neuronal dynamics in the beta and gamma frequency bands.

\section{Materials and Methods}

Experimental design and statistical analysis. The experimental design to test the effects of optogenetic stimulation on coherent neuronal dynamics consists of three parts. First, we built a phenomenological model of optogenetic stimulation-evoked coherent neuronal dynamics. In this model, the E/I response is defined as the optogenetic-stimulation-evoked LFP response. In addition, the E/I response controls the likelihood of spiking. Thus, the E/I response model directly links LFP activity and spiking dynamics. To test whether changes in the E/I response are sufficient for driving different coherent neuronal dynamics, we systematically vary the parameters that control the temporal dynamics of the E/I response and measure the spike-field coherence (SFC) in the simulations.

Next, we empirically test the phenomenological predictions of the E/I response model. We measure both spiking and LFP activity during optogenetic stimulation in awake, alert nonhuman primates (NHP). We measured evoked LFP and spiking responses to single stimulation pulses, as well as coherent neuronal dynamics that result from extended pulse sequences. To verify that the E/I response model captures the features of the empirically observed neural activity, we fit the E/I response using the empirically measured single pulse evoked LFP responses. The fitted parameters are then used in the E/I response model to generate simulated coherent neuronal dynamics for the same conditions as the empirically measured activity.

Finally, we perform simulations of leaky integrate-and-fire neurons with a subpopulation of ChR2-positive neurons and compare the simulated coherent neuronal dynamics with the empirical observed dynamics. Importantly, the spiking network model offers access to EPSCs and IPSCs that could not be measured directly from extracellular electrodes. We use the spiking network model to investigate the mechanisms of the observed optogenetic stimulation-evoked coherent neuronal dynamics.

Animals. Recordings were taken from two male rhesus macaques (Macaca mulatta; Monkey J: 12 years, $6.5 \mathrm{~kg}$; Monkey H: 11 years, 10.5 $\mathrm{kg}$ ) and one male cynomolgus macaque (Macaca fascicularis; Monkey B: 4 years, $5 \mathrm{~kg}$ ). Monkeys $\mathrm{J}$ and $\mathrm{H}$ were part of previous studies using the contralateral intraparietal sulcus and the ipsilateral frontal cortex. Monkey B had not been part of previous experiments. All animals were in normal health and were group housed with other conspecifics at the facility. All surgical and animal care procedures were approved by the New York University Animal Care and Use Committee and were performed in accordance with the National Institute of Health guidelines for care and use of laboratory animals.

Injection surgery. We performed injections of optogenetic viral vectors $6-25$ weeks before recording. Subjects were maintained at a constant depth of anesthesia with isoflurane (1-2\%). Monkeys J and $\mathrm{H}$ were injected in existing craniotomies. In Monkey B, after mobilizing the scalp and other soft tissue, we made craniotomies over motor cortex to provide access for injections. Injections were made through $26 \mathrm{~s}$ gauge stainless steel cannulas (Hamilton) that were inserted through a thinned dura. For each injection, $1 \mu \mathrm{l}$ of AAV5-hSyn-ChR2(h134R)-EYFP was injected at a rate of $0.05 \mu \mathrm{l} / \mathrm{min}$. We made injections at $2-3$ depths spaced $500-750$ $\mu \mathrm{m}$ across $2-4$ cortical sites per animal. We injected at multiple sites simultaneously to increase the total number of injections sites and thus increase the probability of having successful transduction at one or more sites. We waited 20 min between each injection to allow the virus to diffuse through the tissue. After injections, chambers were reclosed with the chamber cap and periodically opened for cleaning and maintenance while the viral vector was expressing.

Optical stimulation. We illuminated the injection site with a $473 \mathrm{~nm}$ Laser (Shanghai Sanctity Laser) connected to a multimode fiber-optic cable (200 $\mu \mathrm{m}$ core, BFL-200, Thorlabs). One end of the fiber-optic cable was terminated with an FC/PC connecter and connected to a collimator (PAFA-X-4-A, Thorlabs) for focusing light from the laser. At the other end of the cable we removed a small amount of buffer with a microstripper (T12S21, Thorlabs) and cleaved it with a diamond knife (S90R, Thorlabs) to ensure a flat edge for minimal power loss.

The fiber-optic cable was placed on top of the thinned dura. The cable was held in the microdrive and placed in the same guide tube as the recording electrode. We did not insert the fiber into the brain to mini- 
mize damage to the neural tissue. Light power at the tip varied from 16 to $20.5 \mathrm{~mW}$. (510-653 $\left.\mathrm{mW} / \mathrm{mm}^{2}\right)$, as measured before each experiment using a power meter (PM100D, Thorlabs). Evoked potentials due to artifacts from the Becquerel effect would have rapid onset or offset of measured responses locked to the timing of the light (Cardin et al., 2010). Light-based artifacts typically have a capacitive charging and discharging profile, which has a rapid onset and offset tightly coupled to the onset and offset of the light. The neural response is slower than the duration of the light pulse and thus continues to increase for a brief period after the light has been turned off. The onset is delayed because the ChR2 channel dynamics are slower than the chemical response and thus the membrane potential does not start to change immediately. The initial response recruits network activity, which has a slow time course. This is particularly evident for short light pulses ( $\leq 5 \mathrm{~ms}$ ). When there is no artifact, the response inflection point occurs after the light is turned off. This is less clear for longer pulse durations ( $>15 \mathrm{~ms}$ ) because adaptation and other properties of channel kinetics can lead to a decrease in the response before the end of stimulation. We visually inspected evoked responses for short duration pulses to ensure that that the onset of the response and the inflection point were not aligned with the times when the light was turned on and off and thus not artifacts from the Becquerel effect. We did not find any evidence of rapid responses in the three subjects used in this study. However, we have seen rapid responses in other subjects. One key distinction is stimulation was done in these prior subjects with the fiberoptic cable directly on the brain, rather than transdurally as in the present study. In the current experiment, the Becquerel effect may have been minimized because we stimulated through the dura, which may have sufficiently scattered/diminished light power by the time it reached the electrode.

Light pulse patterns were generated by proprietary software running in LabVIEW (National Instruments) and converted to analog signals using a DAQ card (USB-6251, National Instruments). Stimulation trials consisted of 1-s-long bursts of square wave pulses. For each block of trials, we controlled three stimulation parameters: pulse duration, pulse rate, and pulse distribution. We varied pulse durations from 5 to $20 \mathrm{~ms}$, pulse rates from 5 to $20 \mathrm{~Hz}$ and used both Poisson and periodic pulse distributions. Importantly, we do not use Poisson stimulation because we believe it matches the temporal properties of the intrinsically generated coherent neuronal dynamics. Rather, because Poisson stimulation has energy across all frequencies it allows us to test the frequency content of the driven response across a range of frequencies.

In rodents, both measurement and simulation have proposed that $\sim 1 \%$ of light power density remains after light has passed through $\sim 1$ mm of brain tissue (Aravanis et al., 2007; Huber et al., 2008; Yizhar et al., 2011). However, primate brain tissue may have slightly different optical properties, thus altering the light transmission. In addition, we stimulated transdurally, which may have additional effects on the light. However, we were able to record purportedly directly driven spiking (low-latency, low-jitter) as far as $1.5 \mathrm{~mm}$ below the cortical surface (data not shown), suggesting that we were activating neurons into layer 3 .

We tested each recording site in pseudorandomly interleaved blocks of recordings. Each block consisted of 30-60 repetitions of $1 \mathrm{~s}$ long sequences of square wave pulses (bursts), with $1-3 \mathrm{~s}$ of no stimulation between each stimulation burst. We held other stimulation parameters (pulse rate, duration, and intensity) constant within each block.

Poisson and periodic stimulation pulse trains were generated in silico using the same pulse duration and rate parameters. Simulations consisted of 1000 trials for each combination of parameters.

Electrophysiology. We recorded neural signals from awake, alert macaques while they were seated, head-fixed in a primate chair (Rogue Research) in a dark, quiet room. Neural signals were recorded on glasscoated tungsten electrodes (Alpha-Omega; impedance: $0.7-0.8 \mathrm{M} \Omega$ at 1 $\mathrm{kHz}$ ) held in a FlexMT microdrive (Alpha Omega). Neural signals were acquired at $30 \mathrm{kHz}$ on an NSpike DAQ system and recorded using proprietary data acquisition software. Neural recordings were referenced to a metal wire placed in contact with the dura and away from the recording site. We recorded driven LFP activity and multiunit spiking in premotor cortex in Monkey B (premotor cortex) and posterior parietal cortex in Monkeys J and $\mathrm{H}$.
E/I response model. We model neuronal spiking according to a conditional intensity function, $\lambda(t)$, which represents the instantaneous firing rate, such that probability of a spike occurring at time $t$ is given by $\lambda(t) \Delta t$ (Brown et al., 2003). In the absence of optogenetic stimulation, we model background spiking activity as a Poisson process with a constant rate, $\lambda_{0}$ (Fig. 1A). We model background LFP activity as a process with increased power at low frequencies; "brown noise". For simplicity, we further assume that background LFP activity is uncorrelated with spiking activity and does not affect the value of $\lambda(t)$. Under this assumption, spontaneous spiking does not exhibit SFC, which may be inconsistent with typical physiology. However, we find that SFC during optogenetic stimulation is several times greater than spontaneous SFC (Hagan et al., 2012; Wong et al., 2016) and thus dominated by the driven activity.

To simulate LFPs using an E/I response model, we generated $\frac{T}{d t}$ samples of Brownian noise as background activity, with $T=1 \mathrm{~s}$ and $d t=1$ $\mathrm{ms}$. The driven component of the LFP consists of the impulse response to stimulation pulses. For each recording site we fit a temporal impulse function, $\Theta(t)$, which represents the time course of the neural response following an impulse input (Adelson and Bergen, 1985), to the empirically measured stimulation pulse evoked LFP response as follows:

$$
\begin{gathered}
\Theta(t)=\beta_{E} E-\beta_{I} I, \\
E(t)=A e^{-A t}\left(\frac{(A t)^{\alpha_{E}}}{\alpha_{E} !}\right), \\
I(t)=A e^{-A t}\left(\frac{(A t)^{\alpha_{I}}}{\alpha_{I} !}\right) .
\end{gathered}
$$

The modeled LFP impulse response, $\Theta(t)$, is the sum the of the individual $\mathrm{E}$ and I responses to stimulation. Measurements of LFP activity preferentially weight contributions of EPSCs and IPSCs on neurons with openfield geometries (Buzsáki et al., 2012; Pesaran et al., 2018). Because inhibitory currents hyperpolarize the membrane potential and oppose the effects of excitatory currents, we force $I(t)$ to have a negative contribution to $\Theta(t)$. Thus, $\Theta(t)$ represents the sum of excitatory and inhibitory currents; the $\mathrm{E} / \mathrm{I}$ response.

The parameters $A, \alpha_{E}$ and $\alpha_{I}$ control the dynamics of the response. The effect of both $\alpha_{E}$ and $A$ can be illustrated by finding the maximum of $E(t)$ as a function of $t$. By setting the derivative of $E(t)$ with respect to $t$ equal to zero, we find that:

$$
t_{\max , E}=\frac{\alpha_{E}}{A} .
$$

Holding $A$ constant while increasing $\alpha_{E}$ results in $E(t)$ that has a longer latency onset and lasts for a longer duration. Conversely, holding $\alpha_{E}$ constant while increasing $A$ results in $E(t)$ that has a shorter latency onset and lasts for a longer duration. The same holds true for $A, \alpha_{I}$, and $I(t)$. Because $A$ is the same for both $E(t)$ and $I(t), \beta_{E}$ and $\beta_{I}$ control the relative contributions of the excitatory and inhibitory components to the E/I response.

For the pure simulations, $A, \beta_{e x c}$ and $\beta_{i n h}$ were set to 1 . To fit empirical $\mathrm{E} / \mathrm{I}$ response, $\beta_{E}, \beta_{I}, A, \alpha_{E}$, and $\alpha_{I}$ were fit using a grid search to minimize the squared error between the modeled response and the empirical response. The parameters $\alpha_{E}$, and $\alpha_{I}$ can only take positive integer values, and $\beta_{E}, \beta_{I}$, and $A$ were limited to positive values. For illustrative purposes, we show the E response preceding the I response but this can be reversed so that I precedes E by $\alpha_{E}>\alpha_{I}$ (Cruikshank et al., 2010; Haider et al., 2013).

We simulated the driven LFP by convolving the site-specific E/I response, $\Theta(t)$, with a sequence of delta pulses determined by the stimulation parameters. The driven LFP was added to the background activity to generate the total simulated LFP activity.

We modeled spiking activity that was coherent with the LFP using a Poisson point process with a conditional intensity function. The conditional intensity function models stochastic spiking governed by a timevarying instantaneous firing rate, $\lambda(t)$ : 


\section{A}

Spontaneous activity
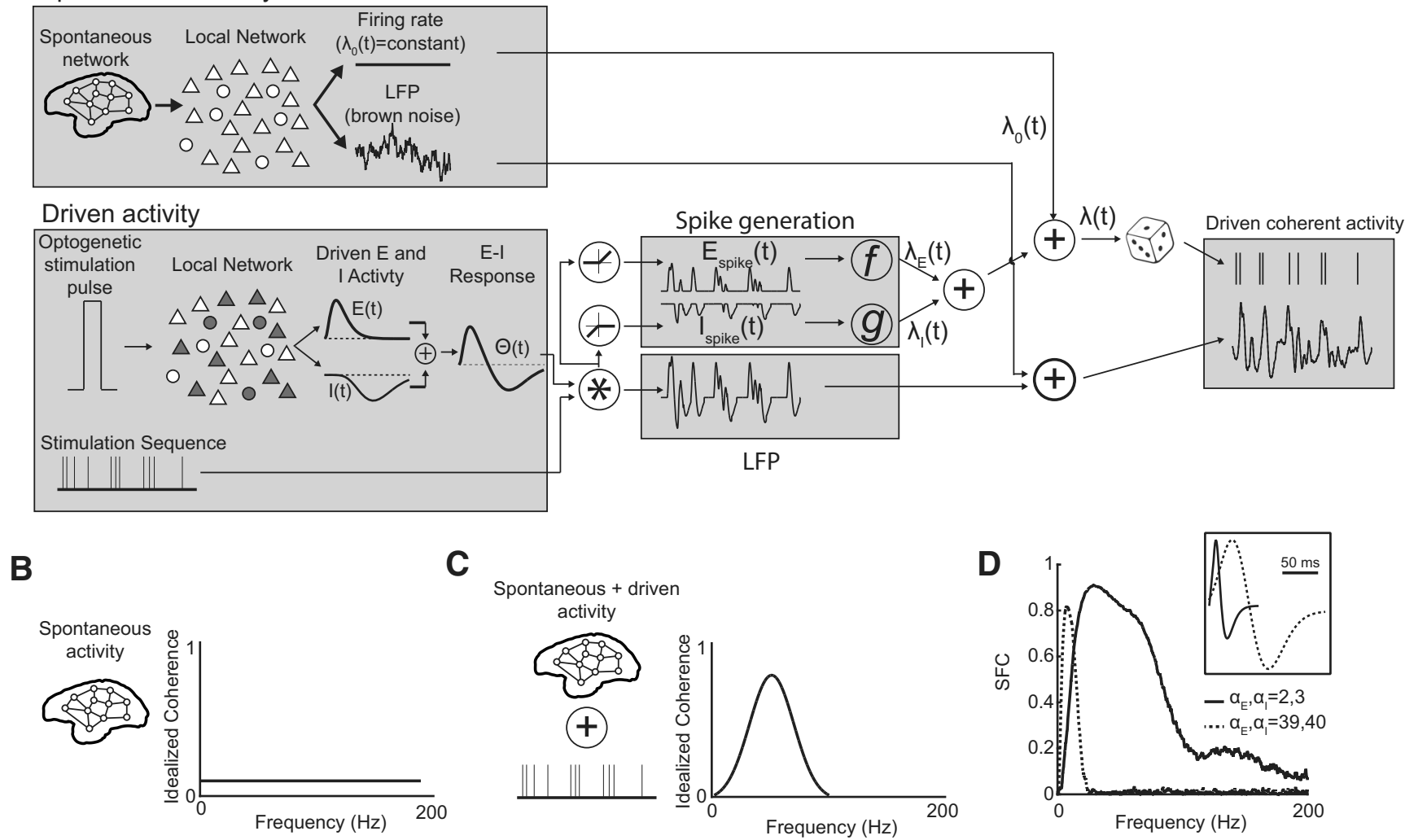

Figure 1. Excitation and inhibition drive coherent neuronal dynamics. $A$, To model optogenetic stimulation of a population of transduced neurons, we simulated pulsatile responses generated by direct activation of ChR2 channels and synaptic activity. Pulsatile responses are convolved with a stimulation sequence. The summed excitatory and inhibitory components, $\Theta(t)$, comprise the simulated LFP. The individual E and I activity govern the variable spiking rate in the Poisson process, which generates coherent spiking. B, Spontaneous activity in the model has no SFC. C, Optogenetic stimulation drives correlated fields and spiking, leading to frequency-selective SFC. D, Simulated SFC for short and long E// responses. Inset, E/I responses used in the simulation.

$$
\begin{gathered}
\lambda(t)=\left(\lambda_{E}(t)+\lambda_{I}(t)+\lambda_{0}\right) \cdot d t, \\
\lambda_{E}(t)=f\left(E_{\text {spike }}(t)\right)=e^{E_{\text {spike }}(t)}, \\
\lambda_{I}(t)=g\left(I_{\text {spike }}(t)\right)=e^{I_{\text {spike }}(t)}, \\
E_{\text {spike }}(t)=\left\{\begin{array}{cc}
\Theta(t), & \Theta(t) \geq 0 \\
0, & \text { otherwise }
\end{array}\right. \\
I_{\text {spike }}(t)=\left\{\begin{array}{cc}
\Theta(t), & \Theta(t)<0 \\
0, & \text { otherwise }
\end{array}\right.
\end{gathered}
$$

where $\lambda_{0}$ is the baseline firing rate, and $d t$ is the sampling rate. $E_{\text {spike }}(t)$ and $I_{\text {spike }}(t)$ are determined by convolving the $\mathrm{E} / \mathrm{I}$ response, $\Theta(t)$, with a Poisson process generated from the stimulus sequence parameters then positively or negatively rectifying to get the separate $\mathrm{E}$ and I responses. Thus, $E_{\text {spike }}(t)$ and $I_{\text {spike }}(t)$ are the time courses of driven $\mathrm{E}$ and I responses to the sequence of stimulation pulses, respectively, that generate the fluctuations in the conditional intensity function, $\lambda(t)$. Individual action potentials are then randomly generated at each time point from a binomial random variable with $p($ spike at time $t)=\lambda(t) \Delta t$. After a spike we used a fixed refractory period by setting $\lambda(t)=0$ for $3 \mathrm{~ms}$.

Simulations of the E/I response model were run using custom scripts in MATLAB using a $1 \mathrm{~ms}$ time step. For each trial, we simulated $1 \mathrm{~s}$ of baseline activity and $1 \mathrm{~s}$ of stimulation. We simulated 1000 trials for set of stimulus parameters.

Spiking network model. The spiking network model consists of a network of 4000 excitatory and 1000 inhibitory leaky integrate-and-fire neurons, each of whose membrane potential, $V$, is described by the following equation:

$$
\tau_{m} \frac{d V}{d t}=-\left(V-V_{r}\right)+V_{A}-V_{G}+\frac{k \tau_{m} I_{C h R 2}}{C_{m}} .
$$

Neurons are randomly connected to each other with a connection probability $P_{e / i, e / i}$. In addition, the neurons receive connections from external inputs (described in External inputs), which represent synaptic input from spontaneous activity in other areas of the brain. The synaptic potentials, $V_{A}$ and $V_{G}$, taken from the Mazzoni model (Mazzoni et al., 2008), represent the sum of synaptic responses of each neuron induced by individual presynaptic spikes from external inputs and other excitatory (pyramidal, AMPA) and inhibitory (interneurons, GABA) neurons in the model.

$$
\begin{gathered}
\tau_{d A} \frac{d V_{A}}{d t}=-V_{A}+x_{A} \\
\tau_{r A} \frac{d x_{A}}{d t}=-x_{A}+\tau_{m}\left(J_{p y r} \sum_{p y r} \delta\left(t-t_{p y r}-\tau_{L}\right)+J_{e x t} \sum_{e x t} \delta\left(t-t_{e x t}-\tau_{L}\right)\right) \\
\tau_{d G} \frac{d V_{G}}{d t}=-V_{G}+x_{G} \\
\tau_{r G} \frac{d x_{G}}{d t}=-x_{G}+\tau_{m}\left(J_{i n t} \sum_{i n t} \delta\left(t-t_{i n t}-\tau_{L}\right)\right)
\end{gathered}
$$

In the model, $t_{\text {pyr } / \text { intlext }}$ is the time of a spike on a presynaptic neuron. The synaptic currents generated by presynaptic spikes have latency $\tau_{L}$ and 
Table 1. Parameters used for simulations using the spiking network model

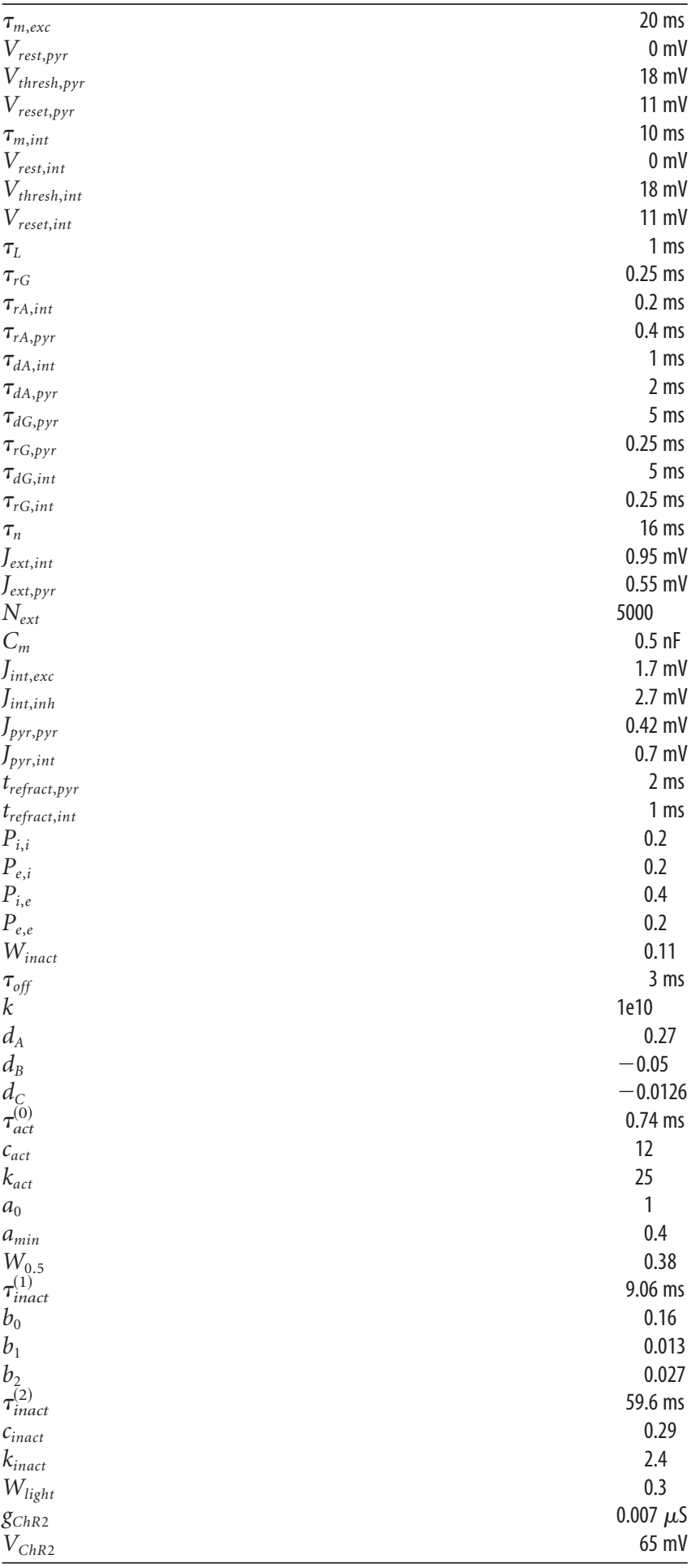

have dynamics governed by their respective exponential rise times $\left(\tau_{r A}, \tau_{r G}\right)$ and decay times $\left(\tau_{d A}, \tau_{d G}\right)$. The efficacy of each connection type is given by $J_{\text {pyr/intlext }}$ and is uniform for each neuron type. Table 1 shows the parameters for the model.

External inputs. We modeled spontaneous external activity as inputs generated from excitatory neurons having random Poisson spike trains with a time-varying rate, $r_{e x t}(t)$, that is the same for all neurons (Mazzoni et al., 2008). The spike trains are generated from a Poisson process with a rate governed by an Ornstein-Uhlenbeck process:

$$
\begin{gathered}
r_{\text {ext }}(t)=\left\{\begin{array}{cc}
c_{0}+n(t), & c_{0}+n(t) \geq 0 \\
0, & \text { otherwise }
\end{array},\right. \\
\tau_{n} \frac{d n(t)}{d t}=-n(t)+\sigma_{n} \sqrt{\frac{2}{\tau_{n}}} \theta(t),
\end{gathered}
$$

where $\theta(t)$ is Gaussian white noise, $c_{0}$ is a non-time-varying mean firing rate and $r_{\text {ext }}(t)$ is half-wave rectified to ensure that all firing rates are non-negative.

Channelrhodopsin photocurrent. We modeled the channelrhodopsin photocurrent in the transduced neurons according to a model from Witt et al. (2013).

$$
\begin{aligned}
& I_{C h R 2}(t)=-a_{\text {refract }} g_{C h R 2} F_{C h R 2}\left(t, W_{\text {light }}\right)\left(V-V_{C h R 2}\right), \\
& F_{C h R 2}\left(t, W_{\text {light }}\right)=A_{\text {act }}\left(1-e^{-\frac{t-t_{O N}-d}{\tau_{\text {act }}}}\right)\left(A_{\text {persist }}+A_{\text {inact }}^{(1)} e^{-\frac{t-t_{O N}-d}{\tau_{\text {inact }}^{(1)}}}\right. \\
& +A_{\text {inact }}^{(2)} e^{\left.-\frac{t-t_{\mathrm{ON}-d}}{\tau_{\text {inact }}^{(2)}}\right),} \\
& d=d_{A}+d_{B} W_{\text {light }}+\frac{d_{c}}{W_{\text {light }}}, \\
& \tau_{a c t}=\tau_{a c t}^{(0)}+c_{a c t} e^{-k_{a c t} W_{l i g h t}}, \\
& A_{\text {act }}=a_{0}+\frac{a_{\min }-1}{1+\left(\frac{W_{0.5}}{W_{\text {light }}}\right)^{2}}, \\
& A_{\text {inact }}^{(1)}=b_{0}+\frac{b_{1}}{b_{2}+\left(W_{\text {light }}-W_{\text {inact }}\right)^{2}}, \\
& A_{\text {inact }}^{(2)}=c_{\text {inact }} e^{-k_{\text {inact }} W_{\text {light }} \text {, }} \\
& A_{\text {persist }}=1-A_{\text {inact }}^{(1)}-A_{\text {inact }}^{(2)} \text {. }
\end{aligned}
$$

The photocurrent, $I_{C h R 2}$, is defined as the product of the conductance, $g_{C h R 2} F_{C h R 2}\left(t, W_{\text {light }}\right)$, and the driving force, $V-V_{C h R 2}$, from the ChR2 channels at any time, $t$. When light stimulation is on, the conductance varies over time as described by the function $F_{C h R 2}$. When the light is off, the conductance returns to baseline following a single exponential decay with a time constant of $\tau_{O F F}$. The light-induced conductance change is described as the product of activation and inactivation functions, which depend on the time from light onset, $t-t_{O N}$, a light intensitydependent delay, $d$, and their respective time constants, $\tau_{\text {act }}, \tau_{\text {inact }}^{(1)}$, and $\tau_{\text {inact }}^{(2)}$. The parameters for Equations 11-19 have been previously fitted to photo conductance measured in vitro human embryonic kidney cells transduced with AAV1/2-CAG-ChR2-YFP (Witt et al., 2013). Table 1 shows the parameters for the ChR2 current part of the model. Each neuron of each type was randomly assigned to have ChR2 channels according to the transduction probability for that cell type. We used a viral vector containing the pan-neuronal hSyn promoter, which has expression rates that are similar across excitatory and inhibitory neurons (Diester et al., 2011). Therefore, we set equal transduction probabilities equal to $1 \%$ for both excitatory neurons and inhibitory neurons.

Previous studies have shown that transduced neurons do not have robust spiking responses to individual light pulses at higher stimulation frequencies (Lin et al., 2009), which is likely because of the slower kinetics of ChR2 channels. This response fidelity drop-off tends to start at $\sim 20$ $\mathrm{Hz}$ periodic stimulation and reaches near zero at $\sim 80-100 \mathrm{~Hz}$ stimulation (Cardin et al., 2009). Therefore, light pulses that occurred $<50 \mathrm{~ms}$ after the previous pulse activated ChR2 channels with a probability that depended linearly such that:

$$
a_{\text {refract }}=\left\{\begin{array}{lc}
1, & \Delta t \geq 50 m s \\
1, & P<\frac{\Delta t}{50 m s}, \\
0, & \text { otherwise }
\end{array}\right.
$$


where $P \in$ unif $(0,1)$ and is chosen individually for each close pulse occurrence and for each model neuron.

Preprocessing in vivo data. Broadband neural activity was split into multiunit signals and LFPs by high-pass and low-pass filtering, respectively, at $300 \mathrm{~Hz}$. LFP signals were downsampled to $1 \mathrm{kHz}$. Multiunit activity (MUA) was computed by applying a $3.5 \mathrm{SD}$ threshold to the multiunit signals to identify putative spikes. All waveforms that exceeded this peak were labeled as multiunit action potentials.

Preprocessing in silico data. LFPs measure currents generated by synaptic activity within a volume near the electrode (Logothetis, 2003). Therefore, we followed Mazzoni et al. (2008) and modeled the LFP as a sum of synaptic currents. Because interneurons typically have dendrites that are organized in a symmetrical, closed-field configuration, we assumed their contribution to the LFP would be minimal compared with synaptic currents on the typically dipole-like dendrites of pyramidal neurons (Buzsáki and Wang, 2012; Pesaran et al., 2018). Therefore, only synaptic currents in pyramidal neurons contributed to our measure of the model LFP. Adding together the absolute values of synaptic currents has been shown to accurately reproduce the power spectrum and information content of LFP recorded in macaque visual cortex (Mazzoni et al., 2008). Simulated LFPs were also low-pass filtered at $300 \mathrm{~Hz}$ and downsampled to $1 \mathrm{kHz}$. Simulated multiunit activity consisted of all the threshold crossings in the leaky integrate-and-fire model, as recorded by the Brian simulator. The in vivo and in silico data were preprocessed, were treated the same after preprocessing.

Data analysis. We computed multiunit peristimulus time histograms (PSTHs) by aligning MUA to the onset time of light pulses. Individual spikes were collected in $1 \mathrm{~ms}$ bins and the corresponding histogram was smoothed by convolution with a Gaussian function with a SD of $1 \mathrm{~ms}$. To avoid corruption from nearby pulses, evoked potentials were computed by averaging LFPs aligned to the onset time of light pulses only for pulses that occurred at the end of a pulse train and did not have a pulse preceding it by $<50 \mathrm{~ms}$.

Spectral analysis was performed using multitaper methods (Mitra and Pesaran, 1999). The accuracy of the spectral quantities we used does not depend on the center frequency of the estimate for all frequencies above the minimum resolvable frequency, given by the frequency smoothing in hertz (Jarvis and Mitra, 2001). For each recording, power spectra were computed using a $1 \mathrm{~s}$ analysis window and $\pm 1.5 \mathrm{~Hz}$ frequency smoothing. We computed power spectra for the LFP responses during entire $1 \mathrm{~s}$ burst (stimulation epoch) and for $1 \mathrm{~s}$ before the burst (spontaneous epoch). Power spectra were computed for neural signals for the spontaneous and stimulation epochs. Recording sites with power spectra that did not exhibit a significant difference were not included in analysis. The exclusion criteria for recording sessions did not depend on the frequency content of the driven LFP response, the desired effect, but rather only that there was any driven LFP response at all. Sites were excluded if there was no change in the LFP power between spontaneous and stimulation periods for the $10 \mathrm{~ms}$ pulse duration condition, which was likely because of the electrode being placed on the periphery of light cone and thus failing to record driven neural activity. We explicitly confirm this in our experiments by moving the electrode and observing driven responses. We computed the spectrogram for each recording using a $\pm 250 \mathrm{~ms}$ analysis window, stepped $50 \mathrm{~ms}$ between windows, with $\pm 2.5 \mathrm{~Hz}$ frequency smoothing aligned to the start of the burst. We computed the SFC for each recording using a $1 \mathrm{~s}$ analysis window with $\pm 3 \mathrm{~Hz}$ frequency smoothing averaged across at least 30 repetitions.

Data availability. Data are available upon reasonable request.

Code accessibility. Software is available in a GitHub repository linked from http://www.pesaranlab.org.

\section{Results}

\section{Modeling optogenetic stimulation-evoked coherent} neuronal dynamics

Coherent neuronal dynamics arise from synchronous, temporally structured neuronal activity. SFC measures coherent neuronal dynamics by estimating the strength of correlations between spiking activity of individual neurons and neural population ac- tivity in the form of simultaneously-recorded LFP activity. When the spike times of a neuron can be predicted from fluctuations in LFP activity at a particular frequency, the spiking is said to be coherent with LFP activity at that frequency. Neurons that fire consistently at a particular phase of LFP activity are coherent with LFP activity. As a result, neuronal coherence is defined by coherent spiking and LFP activity and can be measured by estimating SFC between spiking and nearby LFP activity (Pesaran et al., 2002, 2018).

Figure $1 A$ presents a model of optogenetic stimulationevoked coherent neuronal dynamics and corresponding idealized neural responses. During spontaneous activity, synaptic inputs drive activity in a local network. We model spontaneous spiking activity as being generated from a Poisson process with a constant rate (Brown et al., 2003) and spontaneous LFP activity as being generated from a Brown noise process. Under these assumptions, spontaneous spiking is not coherent with spontaneous LFP activity (Fig. 1A, Spontaneous activity).

Direct activation of transduced neurons leads to excitatory and inhibitory synaptic currents within the local network of neurons. The resulting $\mathrm{E}$ and I currents in postsynaptic neurons occur at different moments in time, and the relative timing of the $\mathrm{E}$ and I currents determines when neurons fire spikes (Higley and Contreras, 2003; Wehr and Zador, 2003; Cruikshank et al., 2010; Haider et al., 2013). Given that excitatory currents depolarize the membrane potential and inhibitory currents hyperpolarize the membrane potential, the cell has the greatest probability of firing when $\mathrm{E}$ is high and I is low. Therefore, we model the impact of optogenetic stimulation on spiking as an excitatory- and inhibitory-dependent temporal change in the probability of the cell firing (Fig. 1A, Spike generation). Thus, the timing of spiking activity depends on the net postsynaptic $\mathrm{E}$ and I consequences of each stimulation pulse. We use the term E/I response to describe the net postsynaptic $\mathrm{E}$ and I resulting from stimulation.

To simulate neuronal activity that exhibits SFC, we need to model the coupling between spiking and LFP activity. LFP activity predominantly reflects summed postsynaptic potentials (Buzsáki et al., 2012; Pesaran et al., 2018). Because the E and I postsynaptic activity drives spiking, we can model the driven LFP as the sum of the driven $\mathrm{E}$ and I responses, thus linking spiking and LFP activity. LFP activity often follows power law scaling, $\frac{1}{f^{b}}$, typically with $b \approx 2$ (Freeman and van Dijk, 1987; Freeman et al., 2000; Miller et al., 2009). Therefore, we add brown noise $\left(\frac{1}{f^{2}}\right)$ background activity to the driven LFP activity to generate the simulated LFP (Fig. 1A, LFP). Using $\frac{1}{f}$ background LFP produced similar results.

We refer to this model of neuronal coherence as the E/I response model. We model spontaneous spiking and LFP as uncorrelated so the spontaneous activity does not exhibit SFC (Fig. 1B). Because optogenetic stimulation evokes E/I responses that result in the likelihood of an action potential occurring varying dynamically, changes in spike probability are coupled to LFP responses. This coupling generates spikes that are coherent with LFP activity (Fig. 1C). Importantly, the E/I response model predicts that the coherence is due to the temporal dynamics, or shape, of the E/I responses. In line with the prediction, SFC varied with the E/I response duration such that longer $\mathrm{E} / \mathrm{I}$ responses generated SFC at lower frequencies (Fig. 1D). In our empirically measured E/I responses, excitatory component dominated the early stage fol- 
lowed by inhibitory component. This is consistent with sensory stimulation, which typically generates an increase in excitation followed shortly by a corresponding inhibitory response (Higley and Contreras, 2003; Wehr and Zador, 2003). However, inhibition may be stronger and have faster rise times than excitation in certain circuits or behaviors (Cruikshank et al., 2010; Haider et al., 2013). This regime would be captured in the E/I response model by setting $\alpha_{E}>\alpha_{I}$.

Note that the neuronal coherence described by the E/I response model depends on predictability between spikes and LFP activity. This construction is consistent with, but does not require, invoking an oscillator or other phase-consistent process. This construction is also consistent using SFC, as opposed to other metrics of phase consistency, to estimate coherent neuronal dynamics. This is because SFC measures linear predictability between spikes and LFP activity. Note also that our approach contrasts with and is complementary to previous work that has used cell-type-specific optogenetic stimulation to generate neuronal coherence.

Many computational models of the mechanisms of coherent neuronal dynamics have focused on simulating periodic, oscillatory activity (Wilson and Cowan, 1972; Börgers and Kopell, 2003; Brunel and Wang, 2003; Keeley et al., 2019). However, empirically observed neuronal coherence is episodic (Burns et al., 2010, 2011; Xing et al., 2012; Feingold et al., 2015; Sherman et al., 2016; Rule et al., 2017), with phase and frequency varying over time, and can be narrow-band or broadband. The E/I response model expands on these prior models to describe manipulations of empirical coherent neural activity patterns, which are not necessarily oscillatory or narrow-band and are episodic. Therefore, our models suggest that the interaction between extrinsic inputs and intrinsic circuit properties can drive episodic dynamics in addition to oscillatory dynamics shown in previous models.

Testing the $\mathrm{E} / \mathrm{I}$ response model with optogenetic stimulation Next, we tested whether optogenetic stimulation generates empirically observed neural coherence according to the predictions of the $\mathrm{E} / \mathrm{I}$ response model. The model suggests that the frequency of driven coherent neuronal dynamics depends on the $\mathrm{E} / \mathrm{I}$ response dynamics, in particular the duration of increased spiking likelihood. Therefore, varying the duration of the stimulation pulse should change the E/I response dynamics, and thus the frequency content of the resulting driven coherent neuronal activity.

To test this hypothesis, we injected AAV5-hSyn-ChR2 (H134R)-EYFP and recorded neuronal responses in three alert macaque monkeys quietly seated in a primate chair. The hSyn promoter results in similar proportions of light-sensitive neurons in both excitatory and inhibitory cell types (Diester et al., 2011). However, because of the greater proportion of excitatory neurons in cortex, stimulation using a pan-neuronal promoter results in non-uniform drive in which the excitatory response to stimulation is greater than the inhibitory response. We recorded in the inferior and superior parietal lobules in Monkeys $\mathrm{H}$ and $\mathrm{J}$ and lateral to the postcentral dimple in Monkey B (Fig. 2A). We observed strong viral expression at injection sites with robust labeling of neurons and neuropil (Fig. 2B). We performed simultaneous in vivo optogenetic stimulation and recording using a fiber-optic cable placed on a surgically thinned dura and adjacent to the intracortical recording electrode (Fig. 2C). Stimulation consisted of 1-s-long sequences of light pulses (stimulation epoch) followed by 1-3 $\mathrm{s}$ of no stimulation (spontaneous epoch; Fig. 2D). Optogenetic stimulation reliably generated neural re-
A

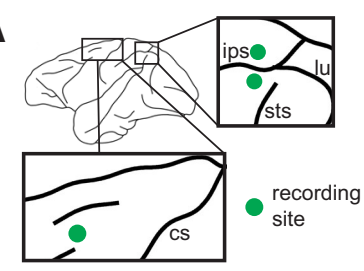

B

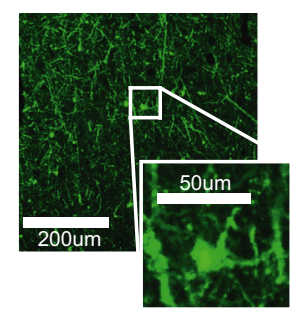

C
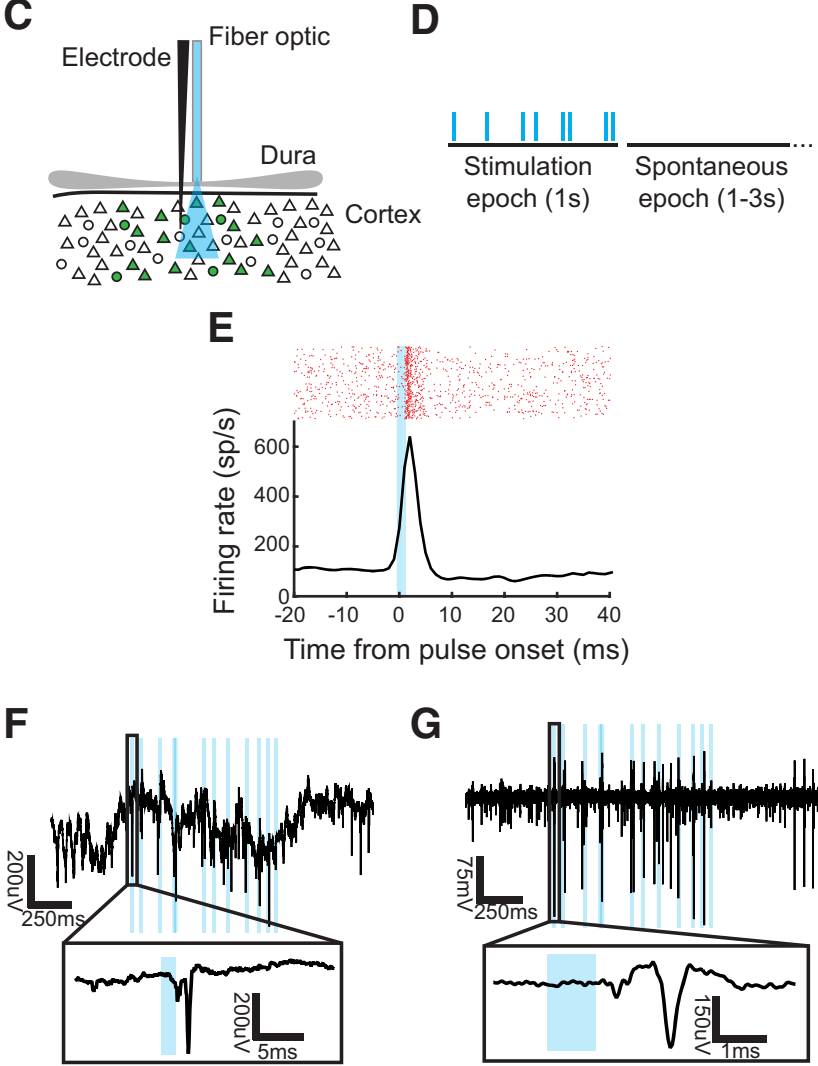

Figure 2. Optogenetic stimulation in macaque cortex. $\boldsymbol{A}$, Recording locations in all animals. $\boldsymbol{B}$, Example injection site with yellow fluorescent protein (YFP) labeled neurons and neuropil from a horizontal section. Inset, Image of a single transduced neuron. $\boldsymbol{C}$, Recordings were performed with a single, acute electrode during trans-dural light stimulation with a fiber-optic cable placed over a surgically thinned dura. D, Experimental design featured a $1 \mathrm{~s}$ stimulation pulse sequence followed by 1-3 s of spontaneous activity without stimulation. $\boldsymbol{E}$, Example raster plot (top) and PSTH (bottom) of the spiking response triggered on each stimulation pulse for Poisson distributed trains of $1 \mathrm{~ms}$ pulses at 10 pulse/s rate. $\boldsymbol{F}$, Example broadband recording during a pulse train containing $1 \mathrm{~ms}$ pulses at 10 pulse/s rate; optogenetic stimulation (blue). Inset, Response to a single pulse. $\boldsymbol{G}$, Example multiunit filtered data from $\boldsymbol{F}$. Inset, Stimulationelicited action potential.

sponses. These responses consisted of action potentials as well as large evoked field potentials visible on an individual pulse basis in the raw data (Fig. $2 E-G$ ).

We measured neuronal responses to stimulation sequences composed of Poisson pulse trains with different pulse rates and pulse durations. Figure $3 A$ presents example neuronal responses during a stimulation block with 5-ms-wide pulses delivered with a $20 \mathrm{pulse} / \mathrm{s}$ Poisson pulse train. Individual stimulation pulses drove spiking activity and evoked LFP responses at the stimulation site (Fig. 3Ai,Aii). We estimated the E/I response, $\Theta(t)$, by fitting the parameters using empirically measured evoked LFP (Fig. 3Aiii). We used this fitted E/I response in the E/I response model to simulate coherent neuronal dynamics. The simulated SFC closely matched the empirically measured SFC (Fig. 3Aiv). 

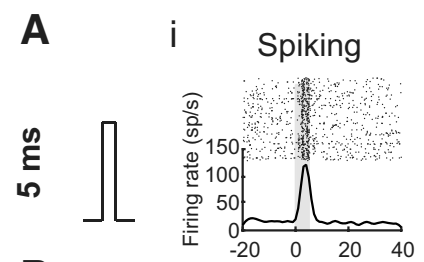

B

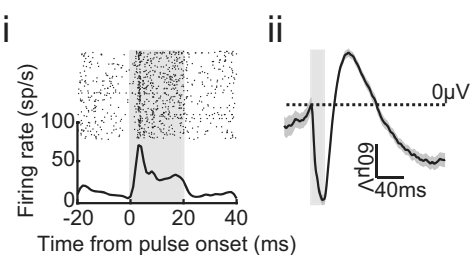

C
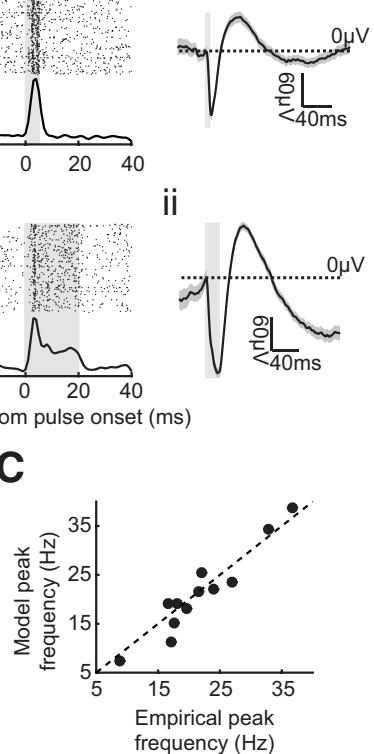

ii Evoked LFP iii Model fit
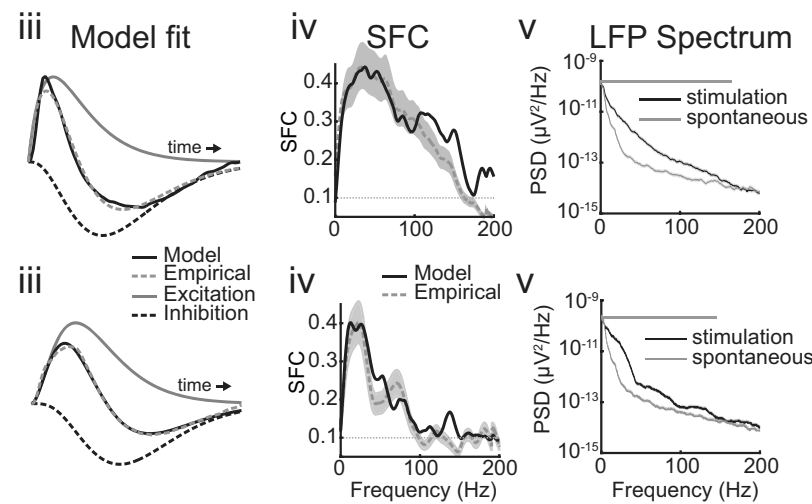

E

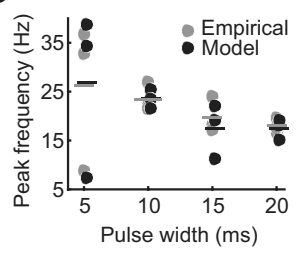

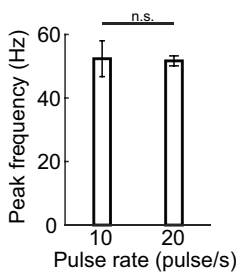

Figure 3. Neuronal dynamics driven by optogenetic stimulation. $\boldsymbol{A}$, Neuronal responses to $5 \mathrm{~ms}$ pulse duration, $20 \mathrm{~Hz}$ pulse rate, Poisson pulse train. Ai, Raster (top) and PSTH (bottom) of the response to single light pulses (gray). Aii, Mean evoked potential for all the stimulation pulses that were the last in a sequence and not preceded by a prior stimulation pulse within $50 \mathrm{~ms}$, normalized by subtracting the value of the LFP at the onset of the light. Dotted line indicates $0 \mathrm{mV}$. Aiii, Modeled E/I response (black) fit to the empirically measured E/I response (gray dotted) with excitatory (gray) and inhibitory (black dotted) components. The fitted E/I response parameters are as follows: A $=0.097, \alpha_{\mathrm{F}}=1, \alpha_{1}=3, \beta_{\mathrm{F}}=2.568, \beta_{1}=3.654$. Aiv, SFC for multiunit activity and LFP measured on the same electrode. The gray area shows the confidence interval $(p=0.01)$ for the empirical SFC, estimated using a Jackknife test. Dotted horizontal gray line shows expected coherence under the null hypothesis that coherence is zero. Av, Power spectral density (PSD) of 1 s of data during the stimulation epoch (black line) and the spontaneous epoch (gray line). The dotted lines are error bars determined by the $\chi^{2}$ test for $p=0.01$. Solid gray lines above the PSD illustrate frequencies where the driven PSD was significantly different from the spontaneous PSD ( $p<$ 0.01). B, Same as $\boldsymbol{A}$ for $20 \mathrm{~ms}, 20$ pulses $/ \mathrm{s}$ pulse rate, Poisson pulse train. The fitted E/I response parameters for $B$ iiii are as follows: $\mathrm{A}=0.097, \alpha_{\mathrm{E}}=2, \alpha_{1}=4, \beta_{\mathrm{E}}=4.548, \beta_{1}=4.730$. $\boldsymbol{C}$, Comparison of the peak frequency of the driven empirical and simulated SFC for all pulse durations conditions and all units. $D$, Peak frequency for the driven SFC for each unit for the empirical data (gray) and E/I response model simulation (black). Horizontal lines show the mean for each condition. E, Average and standard error of the mean (SEM) of the peak of the LFP response for all stimulus sequences with a $10 \mathrm{~ms}$ pulse duration across all recording sites. Differences between means that are not significant are indicated with n.s.

This suggests that the E/I response model captures the phenomenology of the coherent neuronal dynamics driven by optogenetic stimulation. In addition, we computed the power spectrum of LFP activity during the 1-s-long stimulation epoch and for $2 \mathrm{~s}$ of the spontaneous epoch (Fig. 3Av). The stimulation LFP power spectrum revealed a significant increase in power $\left(\chi^{2}\right.$ test, $p<$ $0.01)$.

Longer pulse durations generated longer duration changes in firing rate (Fig. $3 \mathrm{Bi}$ ) and longer periods of excitation and inhibition in the LFP activity (Fig. 3Bii,Biii). The driven SFC was significantly above the theoretical noise floor (Jackknife test, $p=$ 0.01 ) for $0-158 \mathrm{~Hz}$ for the $5 \mathrm{~ms}$ pulse condition (Fig. 3Aiv) and $0-94 \mathrm{~Hz}$ for the $20 \mathrm{~ms}$ pulse condition (Fig. 3Biv). Likewise, the power of the LFP activity was significantly increased ( $\chi^{2}$ test, $p=$ 0.01 ) above baseline for $4-163 \mathrm{~Hz}$ for the $5 \mathrm{~ms}$ pulse condition (Fig. 3Av) and 5-144 Hz for the $20 \mathrm{~ms}$ pulse condition (Fig. 3Bv). Therefore, at the example site longer pulse durations drive more frequency selective increases in SFC and the LFP power spectrum. This shows that, stimulation sequences with different pulse durations can be used to selectively generate coherent neuronal dynamics, consistent with changing the E/I response duration. Thus, the E/I response model predicted the relationship between the temporal properties of the E/I response and the frequency content of the stimulation-evoked coherent neuronal dynamics.

\section{Optogenetic stimulation generates pulse duration- \\ dependent responses}

We computed the SFC for the population of recording sites with driven spiking activity in response to stimulation using Poisson pulse trains with $5,10,15$, and 20 ms duration pulses. For each recording condition, we fit the parameters of the $\mathrm{E} / \mathrm{I}$ response as in Figure 3, $A$ and $B$, and used the E/I response model to generate simulated SFC. For all recordings, the simulated SFC closely matched the empirical SFC (Fig. 3C). In addition, for both the empirical and simulated data, the frequency of the driven SFC varied with pulse duration (Fig. $3 D$ ), consistent with the example site and predictions from the E/I response model. Importantly the center frequency did not depend on the mean pulse rate for Poisson stimulation (Fig. 3E). The decoupling of frequency from pulse rate means that the center frequency of the driven coherence can be controlled without increasing the number of stimulation pulses. Together, these results show that optogenetic stimulation can generate driven coherent neuronal dynamics without the need for an oscillatory or other periodic generator.

Previous work suggests that controlling activity in a single cell type is necessary for driving coherence (Cardin et al., 2009; Sohal et al., 2009; Adesnik and Scanziani, 2010; Lu et al., 2015). However, cell-type-specific stimulation recruits synapticallymediated responses from other strongly connected neurons in the network (Phillips and Hasenstaub, 2016). The frequency content of coherent neuronal dynamics is shaped by the recruited neuronal ensemble (Kato et al., 2017). This suggests that neuronal coherence evoked by cell-type-specific optogenetic stimulation may also drive an effective E/I response. Our empirical results show that pan-neuronal optogenetic stimulation generates $\mathrm{E} / \mathrm{I}$ responses that drive coherent neuronal dynamics. Whether the E/I response model is as effective in predicting the impact of cell-type-specific manipulations that target either E or 
I cells alone is an open question and will likely depend on the strength of recurrent connectivity in the network.

\section{Model-based investigation of excitatory and inhibitory dynamics}

Because we did not have direct experimental access to excitatory and inhibitory currents, we developed a spiking neural network model to study how the frequency content of the driven coherent neuronal dynamics depended on the timing of the $\mathrm{E}$ and I activity. Our objective was to construct a model that exhibited driven SFC with frequency content that was dependent on the stimulation pulse duration in a manner consistent with the empirical data. To do this, we implemented a computational model that consisted of a randomly-connected network of leaky integrate-and-fire excitatory and inhibitory neurons that captures LFP responses to sensory stimulation (Mazzoni et al., 2008). A subset of neurons also expressed ChR2 ion channels (Witt et al., 2013; Fig. 4A, B).

The model generated spiking activity and subthreshold potentials in response to optogenetic stimulation (Fig. 4C,D). To test whether the model responses depended on stimulation pulse duration, we simulated data with the same pulse parameters as in Figure 3 (5-20 ms pulse durations; 20 pulse/s Poisson). The model exhibits pulse duration-dependent $\mathrm{E} / \mathrm{I}$ responses (Fig. $4 E$ ) and frequency selectivity that varies with pulse duration (Fig. $4 F$ ). Consistent with the empirical results, longer pulse durations resulted in a decrease in the center frequency of the driven coherent neuronal dynamics (Fig. $4 F$ ). Therefore, the spiking model captures the key features of the empirically observed optogenetically-driven coherent neuronal dynamics. As with the empirical data and $\mathrm{E} / \mathrm{I}$ response model, the frequency selectivity of the driven SFC is pulse durationdependent.

To examine relationship between synaptically mediated $\mathrm{E} / \mathrm{I}$ responses and coherent neuronal dynamics, we defined LFP activity as the sum of postsynaptic currents on excitatory neurons. However, the ChR2 currents are directly linked to stimulation pulse duration and exhibit similar pulse duration-dependent variance as the postsynaptic-based LFP (Fig. $4 G)$. Therefore, currents through the ChR2 channels in directly stimulated neurons may also contribute to the driven coherent neuronal dynamics. Using the ChR2 currents alone in the definition of LFP activity can result in simulated SFC in

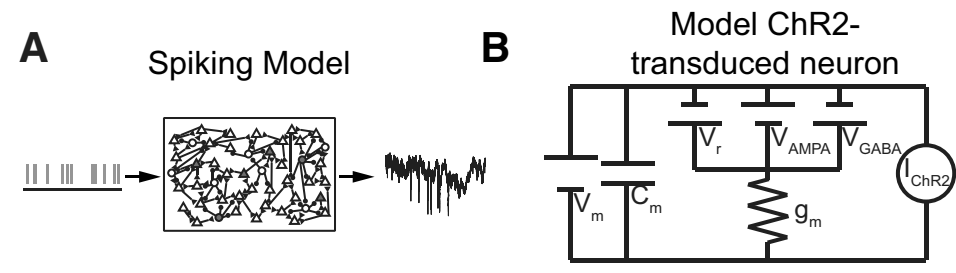

C
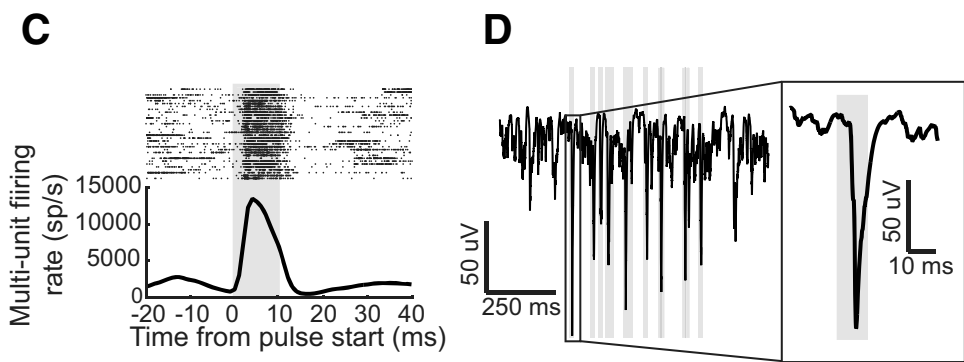

E

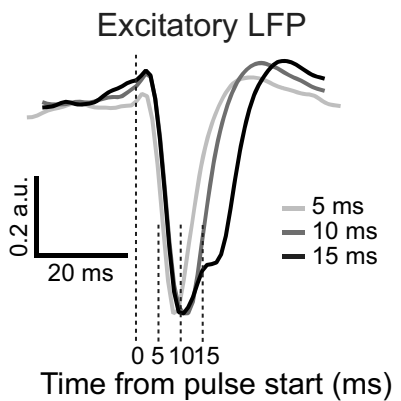

$F$
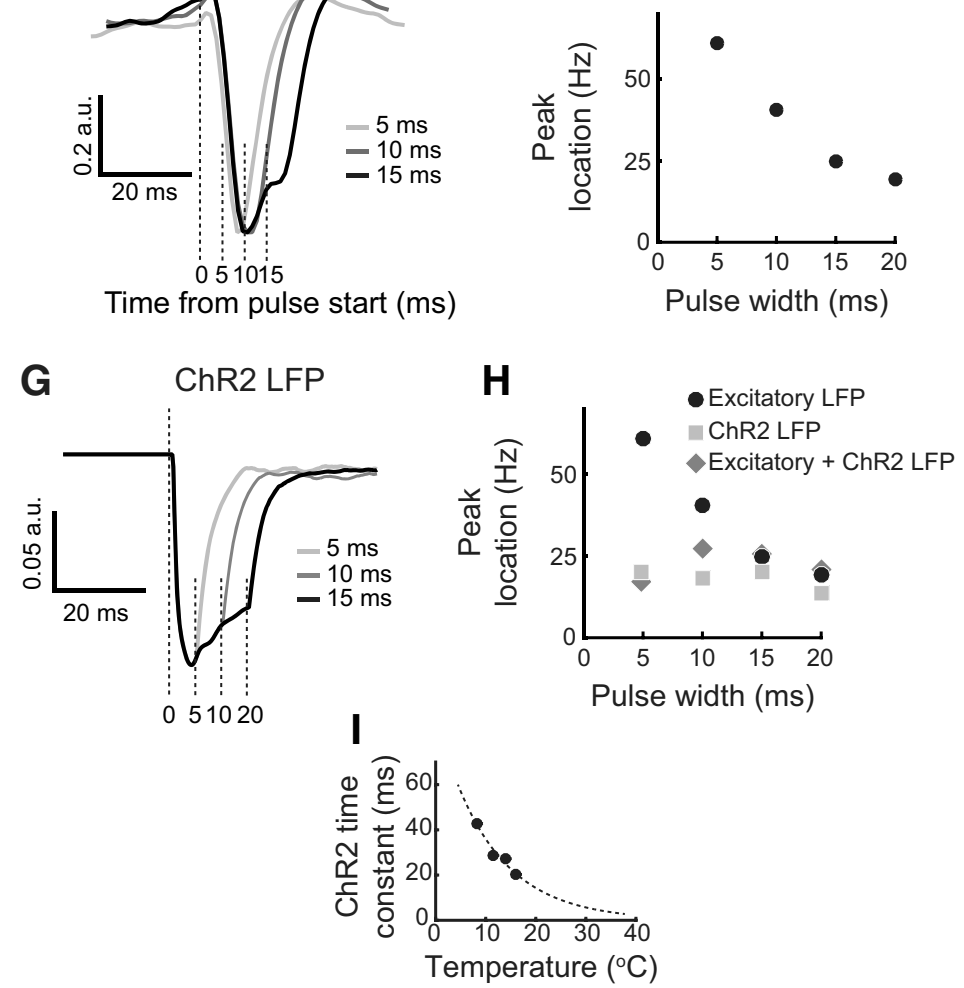

Figure 4. Spiking model responses and dynamics. $A$, Diagram of a leaky integrate-and-fire model network with ChR2transduced neurons. $\boldsymbol{B}$, Circuit diagram of leaky integrate-and-fire neurons with ChR2 channels that make up the spiking network model. C, Raster (top) and PSTH (bottom) of the spiking model response to single light pulses for $10 \mathrm{~ms}, 10$ pulses/s, Poisson stimulation. $\boldsymbol{D}$, Example broadband spiking model simulated LFP activity during a pulse sequence (10 ms, 10 pulses $/ \mathrm{s}$ Poisson stimulation; gray lines indicate pulse times). Inset, Broadband simulated LFP activity at the time of a single pulse. $\boldsymbol{E}$, Mean normalized evoked potentials for the spiking model during pulse trains with $5 \mathrm{~ms}, 10$ and $15 \mathrm{~ms}$ pulse durations and 10 pulses/s, Poisson stimulation. The dotted line at 0 indicates the onset of the light stimulation and the subsequent dotted lines show the time when the light turned off. $\boldsymbol{F}$, Peak frequency of spiking model simulated SFC for each pulse duration. $\boldsymbol{G}$, Mean normalized evoked potentials, with the LFP defined as only coming from the ChR2 currents, for the spiking model during pulse trains with 5, 10, and $15 \mathrm{~ms}$ pulse durations and 10 pulses/s, Poisson stimulation. The dotted light line at 0 indicates the onset of the light stimulation and the subsequent dotted lines show the time when the light turned off. $\boldsymbol{H}$, Peak frequency of spiking model simulated SFC for each pulse duration using different definitions of the LFP. Circle, Postsynaptic currents on excitatory neurons, as in Figure 4F; square, currents from ChR2 channels; diamond, the sum of both postsynaptic currents on excitatory neurons and ChR2 channels currents. I, ChR2 channel kinetics derived from data presented in Feldbauer et al. (2009), modeled with an exponential fit and extrapolated to macaque body temp $\left(37^{\circ} \mathrm{F}\right)$. 
A
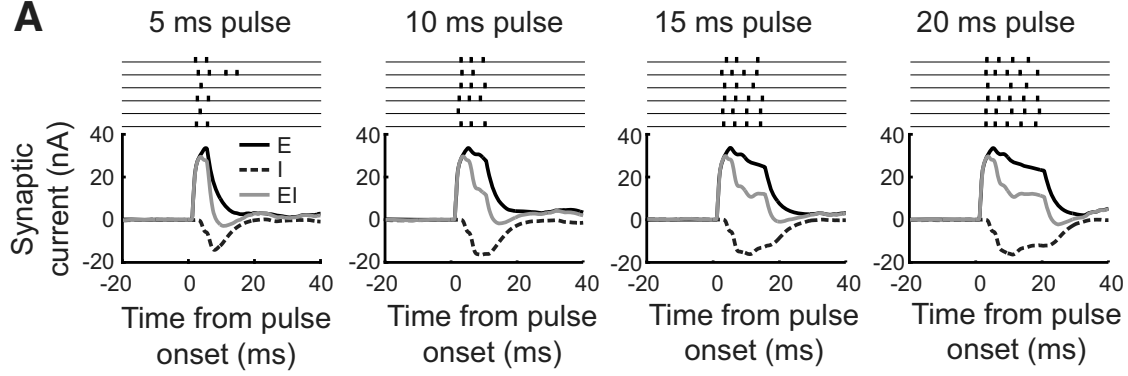

B
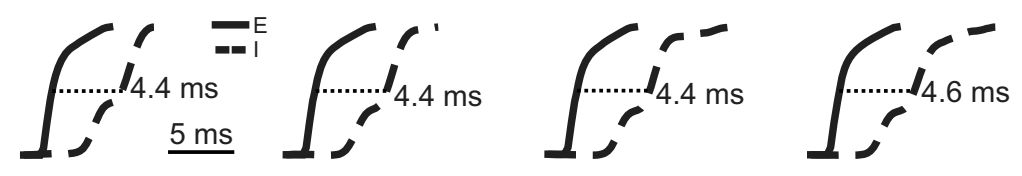

C
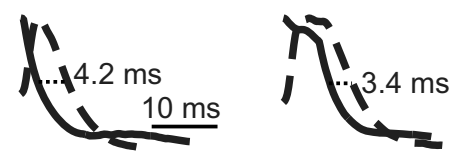

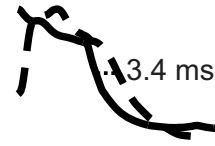

Figure 5. The dynamics of $\mathrm{E}$ and I responses varies with pulse duration. $\boldsymbol{A}$, Stimulation pulse-triggered excitatory (black), inhibitory (dotted), and combined E/I responses (gray) in neurons in the spiking network model in response to 5, 10, 15, and $20 \mathrm{~ms}$ duration simulation pulses. Examples of single pulse evoked spiking are shown above. $\boldsymbol{B}$, Onset of normalized excitatory (solid) and inhibitory (dashed) responses for 5, 10, 15, and 20 ms pulse durations. Onset delay is the difference between the time to half maximal amplitude for excitatory and inhibitory responses. C, Offset for normalized excitatory (solid) and inhibitory (dashed) responses for different pulse durations. Offset delay is the difference between the time to half-maximal amplitude from the peak of the onset response for excitatory and inhibitory responses.

the beta band (Fig. $4 H$, squares). Thus, direct stimulation responses may contribute in part to the empirically observed driven dynamics. However, the center frequency of the ChR2-only SFC does not vary with the pulse duration (Fig. $4 H$, squares). Furthermore, adding ChR2 currents to the postsynaptic currents on excitatory neurons in the LFP definition eliminates the pulseduration dependence for the SFC (Fig. 4H, diamonds). Pulseduration dependence is present when only considering synaptic currents, suggesting that variation in the driven coherent neuronal dynamics depends on postsynaptic E/I responses and is not simply a direct consequence of the simultaneous stimulation excitatory and inhibitory neurons.

The spiking model responses depended on both channel and connectivity parameters. The closing time constant of the ChR2 channel, $\tau_{O F F}$, governed the duration of the response. For longer $\tau_{O F F}$, as by Witt et al. (2013), the duration of the evoked spiking and LFP responses in our spiking network model extended well beyond the end of the light pulse. This was because the ChR2 channels slowly closed after the light turned off, allowing excitatory currents to continue for an extended period of time. To account for the differences in ChR2 and ChR2(H134R) (Lin et al., 2009) channel kinetics and their temperature dependence (Feldbauer et al., 2009) we fit an exponential model to temperaturedependent channel closing times and computed the in vivo closing time constant for ChR2(H134R) as $\sim 3 \mathrm{~ms}$ (Fig. $4 \mathrm{I}$ ). Setting $\tau_{\text {OFF }}$ to $3 \mathrm{~ms}$ generated a response that did not have responses that extended well beyond the offset of the light pulse (Fig. 4C-E).

\section{Driven coherent neuronal dynamics depend on timing of excitatory and inhibitory responses}

The spiking model allows us to study the dynamics of intracellular currents during stimulation. We estimated the mean driven excitatory and inhibitory currents in the neurons in the model by taking the stimulation pulse triggered average of AMPA-mediated currents, $I_{A}$ $=\frac{V_{A} C_{m}}{\tau_{m}}$ and GABA-mediated currents, $I_{G}=\frac{V_{G} C_{m}}{\tau_{m}}$, respectively (Fig. $5 A$ ). Driven excitatory currents are greater than inhibitory currents. As the stimulation pulse duration increases, the driven excitatory and inhibitory currents persist for a longer period of time (Fig. 5A), and the relative timing of the onset and offset of the excitatory and inhibitory currents remains the same, with excitatory currents leading inhibitory currents (Figs. $5 B, C)$. The peak $\mathrm{E}$ current is larger in magnitude than the I current, likely because of the fact that excitatory neurons outnumber inhibitory neurons $4: 1$ in the network. The E current dominated the E/I response, resulting in a stimulationdriven increase in spiking probability. In addition, the duration of time during which $\mathrm{E}$ current dominated the E/I response, varied with pulse duration. Thus the frequency content of the driven coherent neuronal dynamics depends on the duration of the $\mathrm{E}$ and I responses.

Previous work has suggested that balanced inhibition is responsible for the temporal precision of spiking in response to sensory stimulation (Wehr and Zador, 2003). In our spiking network model, E/I balance arises from correlations between excitatory synaptic currents and inhibitory synaptic currents during both spontaneous activity and optogenetic stimulation and is thus an intrinsic property of the circuit (Figs. 6A-C). Consistent with previous experimental results, we find that inhibition lags excitation in the spiking model. Interestingly, the lag decreases during optogenetic stimulation (2.6 ms during spontaneous activity and $1.8 \mathrm{~ms}$ during stimulation; Fig. $6 B$ ) and coherence between $\mathrm{E}$ and I activity increases (Fig. 6D) so that the inhibitory activity more closely tracks the excitatory activity.

The spiking model shows that optogenetic stimulation drives both excitatory and IPSCs in neurons. The excitatory currents precede the inhibitory currents and increase the probability of spiking. However, the network tightly couples $\mathrm{E}$ and I activity, resulting in $\mathrm{E} / \mathrm{I}$ balance. Because of this network property, the excitatory currents are followed closely in time by IPSCs. The inhibitory currents counter the initial excitatory response and suppress the previously increased tendency to fire. Because the $\mathrm{E} / \mathrm{I}$ coupling increases during stimulation, the network property that governs $\mathrm{E} / \mathrm{I}$ balance may be responsible for the temporal precision of the driven spiking and thus may be a mechanism for stimulation-driven frequency selective coherent neuronal dynamics.

\section{Discussion}

Here, we investigate the mechanisms by which optogenetic stimulation of NHP cortical neurons evokes coherent neuronal dynamics. We show that optogenetically-driven neuronal dynamics arise from how extrinsic inputs recruit excitatory and inhibitory responses, the $\mathrm{E} / \mathrm{I}$ response, which follow intrinsic circuit properties and influence spike timing. We developed a phenomenological model to 
predict how the frequency of optogeneticstimulation-driven coherence varies parametrically with stimulation parameters according to the timing of excitatory and inhibitory currents generated by each stimulation pulse. Experimental results from optogenetically-stimulating sites in the posterior parietal and frontal cortex confirmed the model predictions. Furthermore, we developed a biophysical spiking network model using parameters drawn from previous work modeling intrinsic neuronal dynamics and observed stimulation-driven $\mathrm{E} / \mathrm{I}$ responses with a pulse-duration dependence that agreed with the empirical coherent neuronal dynamics. These results suggest that neuronal coherence observed under normal circumstances may not necessarily reflect the presence of an oscillatory generator and that intrinsic E/I balance may also contribute. Overall, our results contribute to the growing literature on neural coherence by relating stimulation-driven neuronal dynamics to E/I balance.

\section{Using driven dynamics to study $\mathrm{E} / \mathrm{I}$ balance in macaques}

Intracellular recordings have shown that balanced excitation and inhibition plays a key role in controlling neuronal activity and computation in rodent species (Shu et al., 2003; Haider et al., 2006). However, because of technical challenges of directly recording E and I currents in primate neurons (Tan et al., 2014), direct evidence of E/I balance remains elusive. Extracellular recordings in primates have shown that activity in excitatory neurons is balanced by activity in inhibitory neurons at the network level (Dehghani et al., 2016), which may suggest that E/I balance at the neuronal level affects computation in primate brains. By using a pan-neuronal viral vector we are able to directly recruit varying amounts of $\mathrm{E}$ and I activity. Using both experimental data and computational simulations, we show that this pan-neuronal stimulation results in a structured $\mathrm{E} / \mathrm{I}$ response that follows the inherent network properties that govern $\mathrm{E} / \mathrm{I}$ balance. The driven coherent neuronal dynamics depend on the temporal properties of the E/I response, which can be controlled by setting the stimulation pulse duration. Together, these results provide converging evidence that $\mathrm{E} / \mathrm{I}$ balance plays a role in neuronal computation in the primate brain.

\section{E/I response mechanisms of driven coherence}

Neurons fire spikes more often during periods of excitatory and inhibitory imbalance, called integration windows. Synchronous excitatory synaptic inputs depolarize cellular membrane potentials and drive spiking activity. Inhibition dominates cortical responses to stimulation (Haider et al., 2013) and regulates the temporal properties of integration windows through temporally lagged responses, which balance excitatory inputs and repolarize membrane potentials (Gabernet et al., 2005). Spontaneous excitatory and inhibitory activity are correlated, with inhibition lagging excitation, suggesting that $\mathrm{E} / \mathrm{I}$ balance may arise from the properties of the neural circuit (Okun and Lampl, 2008). Therefore, both time-lagged, correlated excitatory and inhibitory responses may play a key role in spike timing precision in response to optogenetic stimulation, thus generating coherent neuronal dynamics.

The E/I response model describes how excitatory and inhibitory activity affect spike timing and driven coherent neuronal dynamics. To gain further mechanistic insight into the consequences of imbalances in excitatory and inhibitory activity, we developed a biophysical spiking network model. Experimental agreement with the model predictions reinforces the idea that driven coherent neuronal dynamics depends on a mechanism of spike timing precision involving a temporary increase in excitatory activity that leads to an increase in spiking. This increase in excitation is then followed by an increase in inhibitory activity that reduces the likelihood of spiking. In this way, optogenetic stimulation drives $\mathrm{E} / \mathrm{I}$ responses that influence spike timing and can contribute to coherent neural activity.

Using a spiking network model with parameters drawn from the literature and not fit to the stimulation-driven neural responses, we also observed pulse-duration-dependent E/I responses that were consistent with the empirical results. This suggests the $\mathrm{E} / \mathrm{I}$ response model may also offer a mechanism for how SFC arises intrinsically, in addition to being driven by extrinsic inputs. Several lines of work point to the role of E/I dynamics in SFC observed under normal circumstances. Sensory stimulation drives $\mathrm{E} / \mathrm{I}$ responses that govern spike timing (Wehr and Zador, 2003). Because LFP activity reflects temporally structured fluctuations of local excitation and inhibition (Csicsvari et al., 2003; Hasenstaub et al., 2005; Buzsáki et al., 2012), the intrinsically generated E/I responses are reflected in LFP activity. Thus, $\mathrm{E} / \mathrm{I}$ dynamics may also contribute to coherent neuronal dynamics observed under normal circumstances.

\section{Alternative cell-type-specific mechanisms for frequency selectivity of driven coherence}

We proposed that longer duration pulses extend the E/I response and shift coherent dynamics toward lower frequencies following the same cellular mechanism that generates E/I responses with shorter pulses. This interpretation is supported by our biophysical model, which contains homogenous populations of excitatory and inhibitory neurons. However, the pulse duration-dependent change in the frequency content of the driven coherent neuronal 
dynamics may be due to a shift in the population of inhibitory neurons activated in the network. Following stimulation, excitation may recruit interneuron activity across a range of cell types (Pike et al., 2000; Gloveli et al., 2005; Tukker et al., 2007; Buzsáki and Wang, 2012), but the somatostatin and PV+ subtypes likely play a preferential role in suppressing high-frequency activity (Sohal et al., 2009; Cardin et al., 2010; Pfeffer et al., 2013). In particular, somatostatin interneurons that inhibit PV+ interneurons could reduce gamma activity and thus shift SFC toward lower frequencies. If so, we predict that somatostatin interneurons may show an increase in firing rate after stimulation, and $\mathrm{PV}+$ and excitatory neurons may decrease in firing rate due to the increased inhibition for longer pulses. Additional work is needed to test these alternative mechanisms. Recordings from identified excitatory and inhibitory neurons are necessary to assess the network recruitment of inhibition at a cellular level. Performing this test depends on inhibitory cell-type specificity that has been unavailable in primates but may be possible using novel genetic targeting strategies (Stauffer et al., 2016; El-Shamayleh et al., 2017; Galvan et al., 2017). Alternatively, extensions of our biophysical model to include large-scale, heterogeneous neural networks may offer predictions about cell-type-specific contributions to coherent neuronal dynamics.

\section{Poisson stimulation for studying dynamics}

Continuous and periodic pulse optogenetic stimulation sequences have previously been used generate beta and gamma activity and study their effects on behavior (Cardin et al., 2009; Sohal et al., 2009; Adesnik and Scanziani, 2010; Ni et al., 2016). However, using continuous constant and pulsatile periodic stimulation drives responses at only particular frequencies (Cardin et al., 2009; Adesnik and Scanziani, 2010; Lu et al., 2015), likely related to properties of the local network (Buzsáki and Wang, 2012 ) and thus may vary across brain regions and subjects (Lu et al., 2015; Ni et al., 2016). Previous work has shown that nondeterministic pulse sequences can drive gamma activity (Cardin et al., 2009; Kahn et al., 2013). Our work extends these previous results, showing that coherent neuronal dynamics in either beta or gamma frequency bands can be generated by stimulating the same population of neurons using stimulation pulses of different durations.

\section{Potential bias in estimates of coherent neuronal dynamics from LFP activity}

Extracellular recordings of LFP activity and spiking may be biased toward activity in pyramidal neurons. LFP activity preferentially weights postsynaptic potentials in neurons with separated current sources that generate an open-field geometry and spatially structured synaptic inputs (Pesaran et al., 2018). Neurons that receive spatially uniform inputs have transmembrane currents that tend to cancel, resulting in a small extracellular potential. Neurons with spatially separated synaptic inputs are more likely to form current dipoles and have greater contributions to the extracellular membrane potential. Thus, postsynaptic potentials from neurons with extended dendritic architectures, such as pyramidal neurons, are more heavily weighted by LFP activity. Additionally, pyramidal neurons typically have larger cell bodies than other cortical neurons and thus have more easily measurable action potentials. Therefore, our measurement of both spiking activity and LFP, and thus SFC, may be biased toward contributions from postsynaptic potentials in pyramidal neurons. In addition to LFP activity, alternative decompositions of neural data, such as projections of population activity onto lower dimensional subspaces, may be used to estimate coherence. Such estimates may be more effective at identifying spiking that is coherent with presynaptic and postsynaptic activity in neuronal ensembles containing both open- and closed-field neurons.

In conclusion, we systematically modeled neuronal responses to optogenetic stimulation in NHP cortex. Our empirical results highlight the role of generating specific E/I responses by selecting stimulation parameters, pulse duration, to selectively perturb behaviorally-relevant coherent neuronal activity. Our modeling results show that $\mathrm{E} / \mathrm{I}$ responses that shape the extrinsic coherence are an emergent property of the interactions between populations of neurons and indicate that coherence under normal circumstances can also arise from intrinsic E/I balance. Therefore, optogenetic control of neuronal activity can be useful for understanding mechanisms of coherent neuronal dynamics and how they give rise to complex, flexible behaviors.

\section{References}

Adelson EH, Bergen JR (1985) Spatiotemporal energy models for the perception of motion. J Opt Soc Am A 2:284-299.

Adesnik H, Scanziani M (2010) Lateral competition for cortical space by layer-specific horizontal circuits. Nature 464:1155-1160.

Aravanis AM, Wang LP, Zhang F, Meltzer LA, Mogri MZ, Schneider MB, Deisseroth K (2007) An optical neural interface: in vivo control of rodent motor cortex with integrated fiberoptic and optogenetic technology. J Neural Eng 4:S143-156.

Atallah BV, Scanziani M (2009) Instantaneous modulation of gamma oscillation frequency by balancing excitation with inhibition. Neuron 62:566577.

Börgers C, Kopell N (2003) Synchronization in networks of excitatory and inhibitory neurons with sparse, random connectivity. Neural Comput 15:509-538.

Brown EN, Barbieri R, Eden UT, Frank LM (2003) Likelihood methods for neural data analysis. In: Computational neuroscience: a comprehensive approach (Feng J, ed), pp 253-286. Boca Raton, FL: CRC.

Brunel N, Wang XJ (2003) What determines the frequency of fast network oscillations with irregular neural discharges? I. Synaptic dynamics and excitation-inhibition balance. J Neurophysiol 90:415-430.

Burns SP, Xing D, Shelley MJ, Shapley RM (2010) Searching for autocoherence in the cortical network with a time-frequency analysis of the local field potential. J Neurosci 30:4033-4047.

Burns SP, Xing D, Shapley RM (2011) Is gamma-band activity in the local field potential of V1 cortex a "clock" or filtered noise? J Neurosci 31: 9658-9664.

Buschman TJ, Miller EK (2007) of attention in the prefrontal and. Science 315:1860-1862.

Buzsáki G, Wang XJ (2012) Mechanisms of gamma oscillations. Annu Rev Neurosci 35:203-225.

Buzsáki G, Anastassiou CA, Koch C (2012) The origin of extracellular fields and currents: EEG, ECoG, LFP and spikes. Nat Rev Neurosci 13:407-420.

Cardin JA, Carlén M, Meletis K, Knoblich U, Zhang F, Deisseroth K, Tsai LH, Moore CI (2009) Driving fast-spiking cells induces gamma rhythm and controls sensory responses. Nature 459:663-667.

Cardin JA, Carlén M, Meletis K, Knoblich U, Zhang F, Deisseroth K, Tsai LH, Moore CI (2010) Targeted optogenetic stimulation and recording of neurons in vivo using cell-type-specific expression of channelrhodopsin-2. Nat Protoc 5:247-254.

Chariker L, Shapley R, Young LS (2018) Rhythm and synchrony in a cortical network model. J Neurosci 38:8621-8634.

Cruikshank SJ, Urabe H, Nurmikko AV, Connors BW (2010) Pathwayspecific feedforward circuits between thalamus and neocortex revealed by selective optical stimulation of axons. Neuron 65:230-245.

Csicsvari J, Jamieson B, Wise KD, Buzsáki G (2003) Mechanisms of gamma oscillations in the hippocampus of the behaving rat. Neuron 37:311-322.

Dean HL, Hagan MA, Pesaran B (2012) Only coherent spiking in posterior parietal cortex coordinates looking and reaching. Neuron 73:829-841.

Dehghani N, Peyrache A, Telenczuk B, Le Van Quyen M, Halgren E, Cash SS, Hatsopoulos NG, Destexhe A (2016) Dynamic balance of excitation and inhibition in human and monkey neocortex. Sci Rep 6:23176.

Diester I, Kaufman MT, Mogri M, Pashaie R, Goo W, Yizhar O, Ramakrish- 
nan C, Deisseroth K, Shenoy KV (2011) An optogenetic toolbox designed for primates. Nat Neurosci 14:387-397.

El-Shamayleh Y, Kojima Y, Soetedjo R, Horwitz GD (2017) Selective optogenetic control of Purkinje cells in monkey cerebellum. Neuron 95:51-62.e4.

Feingold J, Gibson DJ, DePasquale B, Graybiel AM (2015) Bursts of beta oscillation differentiate postperformance activity in the striatum and motor cortex of monkeys performing movement tasks. Proc Natl Acad Sci U S A 112:13687-13692.

Feldbauer K, Zimmermann D, Pintschovius V, Spitz J, Bamann C, Bamberg E (2009) Channelrhodopsin-2 is a leaky proton pump. Proc Natl Acad Sci U S A 106:12317-12322.

Freeman WJ, van Dijk BW (1987) Spatial patterns of visual cortical fast EEG during conditioned reflex in a rhesus monkey. Brain Res 422:267-276.

Freeman WJ, Rogers LJ, Holmes MD, Silbergeld DL (2000) Spatial spectral analysis of human electrocorticograms including the alpha and gamma bands. J Neurosci Methods 95:111-121.

Fries P (2005) A mechanism for cognitive dynamics: neuronal communication through neuronal coherence. Trends Cogn Sci 9:474-480.

Gabernet L, Jadhav SP, Feldman DE, Carandini M, Scanziani M (2005) Somatosensory integration controlled by dynamic thalamocortical feedforward inhibition. Neuron 48:315-327.

Galvan A, Stauffer WR, Acker L, El-Shamayleh Y, Inoue KI, Ohayon S, Schmid MC (2017) Nonhuman primate optogenetics: recent advances and future directions. J Neurosci 37:10894-10903.

Gloveli T, Dugladze T, Saha S, Monyer H, Heinemann U, Traub RD, Whittington MA, Buhl EH (2005) Differential involvement of oriens/pyramidale interneurones in hippocampal network oscillations in vitro. J Physiol 562:131-147.

Hagan MA, Dean HL, Pesaran B (2012) Spike-field activity in parietal area LIP during coordinated reach and saccade movements. J Neurophysiol 107:1275-1290.

Haider B, Duque A, Hasenstaub AR, McCormick DA (2006) Neocortical network activity in vivo is generated through a dynamic balance of excitation and inhibition. J Neurosci 26:4535-4545.

Haider B, Häusser M, Carandini M (2013) Inhibition dominates sensory responses in the awake cortex. Nature 493:97-100.

Hasenstaub A, Shu Y, Haider B, Kraushaar U, Duque A, McCormick DA (2005) Inhibitory postsynaptic potentials carry synchronized frequency information in active cortical networks. Neuron 47:423-435.

Higley MJ, Contreras D (2003) Nonlinear integration of sensory responses in the rat barrel cortex: an intracellular study in vivo. J Neurosci 23:10190-10200.

Huber D, Petreanu L, Ghitani N, Ranade S, Hromádka T, Mainen Z, Svoboda $\mathrm{K}$ (2008) Sparse optical microstimulation in barrel cortex drives learned behaviour in freely moving mice. Nature 451:61-64.

Jarvis MR, Mitra PP (2001) Sampling properties of the spectrum and coherency of sequences of action potentials. Neural Comput 13:717-749.

Kahn I, Knoblich U, Desai M, Bernstein J, Graybiel AM, Boyden ES, Buckner RL, Moore CI (2013) Optogenetic drive of neocortical pyramidal neurons generates fMRI signals that are correlated with spiking activity. Brain Res 1511:33-45.

Kato HK, Asinof SK, Isaacson JS (2017) Network-level control of frequency tuning in auditory cortex. Neuron 95:412-423.e4.

Keeley S, Fenton AA, Rinzel J (2017) Modeling fast and slow gamma oscillations with interneurons of different subtype. J Neurophysiol 117: 950-965.

Keeley S, Byrne Á, Fenton A, Rinzel J (2019) Firing rate models for gamma oscillations. J Neurophysiol 121:2181-2190.

Lin JY, Lin MZ, Steinbach P, Tsien RY (2009) Characterization of engineered channelrhodopsin variants with improved properties and kinetics. Biophys J 96:1803-1814.

Logothetis NK (2003) The underpinnings of the BOLD functional magnetic resonance imaging signal. J Neurosci 23:3963-3971.

Lu Y, Truccolo W, Wagner FB, Vargas-Irwin CE, Ozden I, Zimmermann JB, May T, Agha NS, Wang J, Nurmikko AV (2015) Optogenetically induced spatiotemporal gamma oscillations and neuronal spiking activity in primate motor cortex. J Neurophysiol 113:3574-3587.

Mazzoni A, Panzeri S, Logothetis NK, Brunel N (2008) Encoding of naturalistic stimuli by local field potential spectra in networks of excitatory and inhibitory neurons. PLoS Comput Biol 4:e1000239.
Miller KJ, Sorensen LB, Ojemann JG, den Nijs M (2009) Power-law scaling in the brain surface electric potential. PLoS Comput Biol 5:e1000609.

Mitra PP, Pesaran B (1999) Analysis of dynamic brain imaging data. Biophys J 76:691-708.

Ni J, Wunderle T, Lewis CM, Desimone R, Diester I, Fries P (2016) Gammarhythmic gain modulation. Neuron 92:240-251.

Okun M, Lampl I (2008) Instantaneous correlation of excitation and inhibition during ongoing and sensory-evoked activities. Nat Neurosci 11:535-537.

Pesaran B, Pezaris JS, Sahani M, Mitra PP, Andersen RA (2002) Temporal structure in neuronal activity during working memory in macaque parietal cortex. Nat Neurosci 5:805-811.

Pesaran B, Vinck M, Einevoll GT, Sirota A, Fries P, Siegel M, Truccolo W, Schroeder CE, Srinivasan R (2018) Investigating large-scale brain dynamics using field potential recordings: analysis and interpretation. Nat Neurosci 21:903-919.

Pfeffer CK, Xue M, He M, Huang ZJ, Scanziani M (2013) Inhibition of inhibition in visual cortex: the logic of connections between molecularly distinct interneurons. Nat Neurosci 16:1068-1076.

Phillips EA, Hasenstaub AR (2016) Asymmetric effects of activating and inactivating cortical interneurons. eLife 5:e18383.

Pike FG, Goddard RS, Suckling JM, Ganter P, Kasthuri N, Paulsen O (2000) Distinct frequency preferences of different types of rat hippocampal neurones in response to oscillatory input currents. J Physiol 529:205-213.

Rule ME, Vargas-Irwin CE, Donoghue JP, Truccolo W (2017) Dissociation between sustained single-neuron spiking and transient $\beta$-LFP oscillations in primate motor cortex. J Neurophysiol 117:1524-1543.

Salkoff DB, Zagha E, Yüzgeç Ö, McCormick DA (2015) Synaptic mechanisms of tight spike synchrony at gamma frequency in cerebral cortex. J Neurosci 35:10236-10251.

Sherman MA, Lee S, Law R, Haegens S, Thorn CA, Hämäläinen MS, Moore CI, Jones SR (2016) Neural mechanisms of transient neocortical beta rhythms: converging evidence from humans, computational modeling, monkeys, and mice. Proc Natl Acad Sci U S A 113:E4885-E4894.

Shu Y, Hasenstaub A, McCormick DA (2003) Turning on and off recurrent balanced cortical activity. Nature 423:288-293.

Sohal VS, Zhang F, Yizhar O, Deisseroth K (2009) Parvalbumin neurons and gamma rhythms enhance cortical circuit performance. Nature 459:698-702.

Stauffer WR, Lak A, Yang A, Borel M, Paulsen O, Boyden ES, Schultz W (2016) Dopamine neuron-specific optogenetic stimulation in rhesus macaques. Cell 166:1564-1571.e6.

Tan AY, Chen Y, Scholl B, Seidemann E, Priebe NJ (2014) Sensory stimulation shifts visual cortex from synchronous to asynchronous states. Nature 509:226-229.

Tukker JJ, Fuentealba P, Hartwich K, Somogyi P, Klausberger T (2007) Cell type-specific tuning of hippocampal interneuron firing during gamma oscillations in vivo. J Neurosci 27:8184-8189.

Veit J, Hakim R, Jadi MP, Sejnowski TJ, Adesnik H (2017) Cortical gamma band synchronization through somatostatin interneurons. Nat Neurosci 20:951-959.

Wehr M, Zador AM (2003) Balanced inhibition underlies tuning and sharpens spike timing in auditory cortex. Nature 426:442-446.

Wilson HR, Cowan JD (1972) Excitatory and inhibitory interactions in localized populations of model neurons. Biophys J 12:1-24.

Witt A, Palmigiano A, Neef A, El Hady A, Wolf F, Battaglia D (2013) Controlling the oscillation phase through precisely timed closed-loop optogenetic stimulation: a computational study. Front Neural Circuits 7:49.

Womelsdorf T, Fries P, Mitra PP, Desimone R (2006) Gamma-band synchronization in visual cortex predicts speed of change detection. Nature 439:733-736.

Wong YT, Fabiszak MM, Novikov Y, Daw ND, Pesaran B (2016) Coherent neuronal ensembles are rapidly recruited when making a look-reach decision. Nat Neurosci 19:327-334.

Xing D, Shen Y, Burns S, Yeh CI, Shapley R, Li W (2012) Stochastic generation of gamma-band activity in primary visual cortex of awake and anesthetized monkeys. J Neurosci 32:13873-13880a.

Yizhar O, Fenno LE, Davidson TJ, Mogri M, Deisseroth K (2011) Optogenetics in neural systems. Neuron 71:9-34. 